\title{
The Origin of Remote Symptoms in Electrical and Lightning Injuries: An Attempt at Explanation and a Hypothesis for Testing
}

\author{
Christopher J. Andrews* \\ 7 Morningview St., Chapel Hill, QLD A069, Australia
}

\begin{abstract}
A hypothesis is proposed for the causation of remote injuries from Electrical and Lightning Injuries(ELI). This theory takes instruction from recent developments in biological psychiatry and in particular the cortisol theory of depression. This is reviewed and its consequences are given.

The theory is developed as follows. Cortisol is a known stress hormone, and is likely to be activated by the trauma of an electrical injury. Intense trauma chronically affects the control mechanisms for cortisol production leading to chronic cortisol level excess. Cortisol damages the areas of the brain involved in the memory, learning, and fluency dysfunctions seen in ELI, and leads to loss of volume of those regions, due to cell death. Cortisol levels affect the production of BDNF - Brain Derived Neurotropic Factor - in a proportion of individuals. BDNF exists in polymorphic forms and BDNF in one form can be severely depleted by stress. The brain loses its plasticity as a result. Depression results, and is of a type consistent with ELI.
\end{abstract}

It is hoped this theory will lead to experiments to support it, and will guide the use of medication for the injury.

Keywords: ELI remote symptoms, ELI psychological hypothesis, Cortisol, BDNF, ELI psychological syndrome, Hippocampus.

\section{INTRODUCTION}

Lightning and Electric Injuries share one feature in common, and this feature is often a stumbling block to those who do not understand the injuries. The injury certainly can show symptoms and signs in the line of current passage, and these can be attributed simply to injury from the current itself. But there are a significant group of symptoms that are psychological in nature, and these cannot be attributed to current passage in all but the rarest case. Indeed the writer sees many injured people, and reads "expert" opinions on their condition, which state that their psychological manifestations are manufactured and represent malingering. The opinion is that if there is no evidence of current traversing the brain, then the injury is not real, or not attributable to an electrical mechanism. In essence that injury is confined to the line of the current only.

These views fail to recognise the fact that the psychological syndrome is well described [1-10] and is consistent between victims who have had no chance of colluding or manufacturing their symptoms.

The author regards it as "the holy grail" of electrical and lightning injuries ("ELI") to discover the mechanism underlying these symptoms - often termed "remote symptoms" - in order to understand and treat them. Indeed it has been suggested that these injuries are the cause of much more suffering, not to mention loss of work, than any other [11]. The author would prefer to see the totality of the injury referred to as a "Post Electric Shock Syndrome".

*Address correspondence to this author at the 7 Morning View St., Chapel Hill, QLD A069, Australia; Tel: + 6173878 5594; Fax: + 61738782842;

E-mail: and.c@pobox.com

\section{REMOTE INJURIES}

The injuries that are seen as the remote group of injuries are set out below [12].

Memory Disturbance $\quad 71 \%$

Concentration Disturbance $\quad 63 \%$

Aggression and Irritation $\quad 67 \%$

Wariness and Phobia $\quad 58 \%$

Loss of Mental Powers $\quad 50 \%$

Social Isolation $\quad 38 \%$

Sleep Disorder $\quad 38 \%$

and others including confusion, word finding disability, anxiety, depression, and learning disorder

An important part of evaluating a victim is to submit them to neuropsychological testing. The aim of such testing, in part, is to objectify the dysfunctions that are seen, and if possible, subdivide them more specifically. In the cohort reported by the author [2], this testing was undertaken and demonstrated the existence of

Memory and Learning Deficits

$\begin{array}{cc}\text { Globally } & 19 \% \\ \text { More specifically } & \\ \text { Visual } & 35 \% \\ \text { Visuospatial } & 38 \% \\ \text { Auditory } & 62 \% \\ \text { erbal Learning Deficit } & 54 \% \\ \text { Verbal Fluency deficit } & 46 \% \\ \text { Concentration and Attention Deficit } & 46 \% / 42 \% \\ \text { Executive Function Deficit } & 38 \% \\ \text { Reduced Executive Speed } & 62 \%\end{array}$

and others including General and Verbal IQ decrease, Dynamic Coordination decrease, Slowed information processing, Deficit in fine motor skills, and anxiety and depression. 
Often neuropsychological testing gave rise to the comment, that these deficits were more often seen in "organic" rather than "functional" conditions. The distinction is important and the terminology will be examined below. The strong appearance of auditory and verbal memory and learning deficits, (these are often termed "spatial" deficits) supports an organic origin to the deficits.

The enigma raised therefore is how brain injury, remote from the passage of electric current could occur and this paper hypothesizes on this question (see also for example [13]).

Electrical and lightning injuries produce physical symptoms of Pain, Fatiguability, and Weakness, as well as the remote symptoms above. The incidence of these was incidentally found to be:

$\begin{array}{ll}\text { Pain } & 71 \% \\ \text { Fatiguability and Weakness } & 7 \% / 4 \% \\ \text { Headache } & 50 \%\end{array}$

and others like clumsiness and incoordination, sensory disturbance, and autonomic disturbances. These are disabling for a long period after the injury also.

The aim of this paper is to setup a plausible hypothesis for how remote injuries may occur. It is fully admitted that this hypothesis is speculative and has not been tested. Nonetheless it is not without some potential support.

In considering general mechanisms for remote injury causation, we might include two major general groups of theories:

1. A neurohumoural mechanism, where the injury causes peripheral release of some neuroactive substance, which in turn acts on the brain.

2. A reverse conduction mechanism. It is known that peripheral activation of pain receptors activates a complex set of interactions at spinal cord level (turning acute pain into a chronic pain syndrome where "Pain is the Disease" rather than its cause), and reverse conduction superiorly via the cord to the brain.

3. and perhaps others.

The author wishes to explore the first of these, and suggests that, in fact, we do not necessarily have to search for a hitherto unknown chemical mediator. Insights in other areas, especially in neuropsychiatry and modern views of the causation of depression, may assist in the formulation.

\section{TERMINOLOGY AND UNDERLYING CONCEPTS}

\subsection{Organic versus Functional Causation}

The terms "organic" and "functional" are terms that the reader will often see with regard to causation of psychological disorders. They will be used in this paper also. In one sense they derive from historical views of theories of mental dysfunction, and with the benefit of current knowledge, are not as clear cut as once thought. The brief discussion here will start from an older viewpoint to make the distinction clearer.
There have been, in the past, two relatively clear-cut circumstances in which mental illness has been thought to occur.

First, an "organic" cause. It is quite obvious that a neurological disease or demonstrable brain disturbance can show emotional consequences. For example, multiple sclerosis, brain tumours, and other changes in brain structure can produce psychological symptoms. Lay people often term such changes as evidence of "brain damage", in the sense that they are observable when recourse if made to investigations of body structure, e.g., CT scans, ultrasound, MRI scans, and so on.

On the other hand, "functional" illness was identified as a dysfunction for which such an organic causation could not be identified. Sometimes the cause was seen not as residing in the individual themselves, for example, it may well have been seen as a psychological reaction which was more a reaction to circumstances than a malady with identifiable cause. Other examples include psychological disturbance as a result of malfunction of other body chemicals, for example the psychological consequences of thyroid disease.

The distinction nowadays is artificial. Even with those maladies we might have called functional, refinements in measuring and observational techniques do identify biochemical changes which are not observable on structural investigations. So functional illness may be just as organic, with the causative process simply being microscopic.

This also brings to mind the distinction between structural and functional investigation modalities.

Structural investigations are just that - they demonstrate structure. These include XRays, MRI scans, CT scans, ultrasound, and so on.

In Electrical Injury and Lightning Injury, (ELI), these are usually normal.

Functional investigations aim to demonstrate chemical and biochemical activity, for example nuclear scans and the like. Hints at abnormalities for ELI victims can be demonstrated in these. These include, for example, deriving an image of the distribution of a radioactive natural body chemical and any abnormality in its distribution.

There is however one caveat. If a functional investigation is to demonstrate abnormality, it must "look" in the place where the damage exists. For example, this author would suggest that the major neuro-muscular abnormality in ELI exists at the level of the motor end plate (the locus where nerve connects to muscle to activate it and fatigue of which can well be seen as the typical muscle fatigue of the ELI victim). Traditional Nerve Conduction Studies are normal, however it should be remembered that these test the function of the major nerve trunks, and not the endplates.

Similarly the sensory transducers are microscopic fine structures in and around skin and major organs. There is little in the way of investigation to test these.

It is not surprising then that gorss investigations in ELI are normal. 


\subsection{Depression}

Depression is a disorder which, in the older view, we could determine as being either organic or functional depending on perceived causation.

Depression is not just "feeling blue". It covers a multitude of symptoms in addition to the mood disturbance. It includes loss of volition, loss of concentration, loss of memory, loss of enjoyment, and loss of initiative, along with sleep disorder, appetite disturbance, weight loss, disturbance in pain sensation, disturbed sexual ability, among several others. A symptom often overlooked is a testiness and badtempered-ness - "bitchiness" - "a short fuse". Further there are particular types of anxiety often associated with depression, and these include specifically the social anxieties and the phobic anxieties. It can include the learning, memory, and fluency features shown above.

A long established theory of depression maintains that neurotransmitters, which mediate the chemical signaling between nerve cells, are depleted in concentration at the signaling point (the "synapse"). These are mono-amine chemicals, and the aim of treatment is to restore their levels.

Returning to causation, depression can result from "organic" causes - brain tumours, and neuronal disorders and degenerations, to include but a few.

Nonetheless, historically there have been many (probably the majority) of depressions for which no structural disease was evident, and these were considered to be functional. Often they may be have been regarded as a reaction to environmental factors. It has been shown that mono-amine deficiency is still exhibited in these cases, making the distinction between organic and functional illness very fuzzy. In the functional case however, it still begs the question of why the monoamine levels have become diminished in the first place.

Of passing interest is the observation that tumours distant from the brain can cause a marked depression, and pancreatic malignancies are strong in this regard. This raises the interesting observation that there must indeed be some communication from periphery to the brain, not completely understood. Hence the old dictum, that if an older patient presents for the first time with a mental illness at a more mature age, they must first be investigated fully for other disease before mental illness.

Recent depression research has implicated other chemicals in the genesis of depression, particularly the stress hormone cortisol and its production pathway and its interaction at neuronal level. It is this observation that gives rise to the hypothesis in this paper.

\subsection{The HPA Axis}

Cortisol is a hormone which controls many body functions. It is a hormone particularly involved in the body stress response. It is produced in the adrenal gland, which is a small gland sitting on the top pole of each kidney.

The adrenal production of cortisol is stimulated by a hormone ACTH (adreno-cortico-trophic-hormone) which is released by the pituitary gland at the base of the brain. An increase in ACTH increases the output of cortisol and vice versa.
The pituitary gland produces ACTH on "command" from the hippocampus, which is a discrete region of the brain. The "command" is given by CRH (Cortico-trophin-releasing hormone), which is released from the hypothalamus. "CRH has been localized to the paraventricular nucleus (PVN) of the hypothalamus, which projects to the median eminence and other hypothalamic and midbrain targets. The CRH gene exists in cellular DNA and therefore there is some genetic element in its expression and production (as is also seen in a family predisposition to depression). Recently, a family of CRH-related peptides, termed the urocortins, has been identified. These peptides probably play a role in integrating multiple aspects of the stress-response, although their functions are largely unknown" [14].

Signalling for the production of cortisol commences from areas having an influence on the hypothalamus (including the area termed the limbic system, of which one component is the hippocampus). CRH is released in the hypothalamus, and it acts on the pituitary which produces $\mathrm{ACTH}$, which in turn acts on the adrenal to produce cortisol. Cortisol is thus highly inducible by stress. The level of cortisol is partly controlled by negative feedback to various parts of this axis.

\subsection{The Action of BDNF}

BDNF (Brain Derived Neurotropic Factor) is an important chemical in maintaining cell growth and connections in the hippocampus especially. It is a protein produced from synthetic processes which transcribe the BDNF gene in cells supporting neurons in the brain. It's physiology is complex and not yet well understood [15].

Concurrent levels in normal subjects indicate that BDNF and cortisol adopt the same circadian rhythm, positively correlated [16].

\subsection{The Hippocampus Hypothalamus and Limbic System}

Although the exact inputs to the hypothalamus are not known, these neurons are largely controlled by serotoninmediated input from the amygdala and hippocampus of the limbic system [14]. It is interesting to note, again in passing, that serotonin is one of the key mono-amines depleted in depression. The restoration of serotonin levels is the function of one set of anti-depressant medications.

It is important to note that the limbic system is a system of the brain, which includes the hippocampus and the amygdala, and is strongly involved in emotional responses, as well as memory and learning. Damage to these areas will therefore affect these functions.

A good description of limbic system function is found in the following:

Those who research clinical depression have been interested in a particular part of the brain called the limbic system. This is the area of the brain that regulates activities such as emotions, physical and sexual drives, and the stress response. There are various structures of the limbic system that are of particular importance. The hypothalamus is a small structure located at the base of the brain. It is responsible for many basic functions such as body temperature, sleep, appetite, sexual drive, stress reaction, and the regulation of other activities. The hypothalamus also 
controls the function of the pituitary gland which in turn regulates key hormones. Other structures within the limbic system that are associated with emotional reaction [and also memory and learning] are the amygdala and hippocampus [17].

\subsection{Depression in ELI}

The author proceeds from the point that depression in ELI is organic in origin. That is, there is some mechanism for the injury to affect the brain to produce the dysfunction seen. The author is swayed by reports from neuropsychologists who opine that the symptoms that they say are similar to those seen in organic injury. Further the author is also swayed by the commonality between some hundreds of victims of ELI that he has seen without any chance of collusion between them.

There will always be a debate however given that there are those who will say that the depression seen in ELI is purely functional and by implication reactive. Indeed they will say that the symptoms claimed above can be seen in any depression whether caused by something deriving from the injury itself, or simply as a reaction to it.

The psychoanalyst might say that the common feature of all depression is loss of some kind. If this is accepted as true, however, a victim of ELI has every reason to appreciate loss keenly, if only from the marked persistent physical symptoms they suffer. In this context loss of function is profound, and there is every reason for depression of a functional kind.

In the end it is of little matter. Even in functional depression chemical changes within the body may be seen, whatever they are ultimately discovered to be.

And in particular, the ongoing physical disturbance will constitute a distress of the highest level, and result in the chronic production of stress hormones like cortisol, which is important in the hypothesis below.

\section{RECENT DEPRESSION RESEARCH}

Strong evidence has been advanced that there is cellular destruction in the hippocampus as a result of depression, remembering incidentally that the hippocampus is strongly responsible for the learning and memory deficits associated with ELI. MRI evidence strongly supports the loss of volume of the hippocampus in depression, in the presence of otherwise normal brain size.

Patients with depression had a statistically significant $19 \%$ smaller left hippocampal volume than comparison subjects, without smaller volumes of comparison regions (amygdala, caudate, frontal lobe, and temporal lobe) or whole brain volume [18].

The loss of hippocampal volume is thought to be a consequence of depression rather than a prescursor to it [19]. A meta-analysis of studies confirms the loss [20]. In addition, there is suggestion that the atrophy is proportional to the length of the depression and may not be reversible [19]. It is also known cortisol is cytotoxic and that the atrophy is caused by cortisol toxicity $[18,19,21]$ (Truly it may be said that untreated depression causes brain damage).
Stress-induced structural remodelling in the adult hippocampus, involving debranching and shortening of dendrites and suppression of neurogenesis, provides a cellular basis for understanding the impairment of neural plasticity in the human hippocampus in depressive illness [22]. It was particularly noted that production of granule precursor cells was decreased, and this occurred after an initial application of only 7 days of stress.

Plasticity refers to the ability of the brain to generate and repair itself in a plastic rather than fixed way. The link suggested is that BDNF is needed for nerve cell regeneration and repair, i.e. plasticity and plasticity is the key loss in depression. In the normal person, cortisol is accompanied by a rise in BDNF promoting plastic repair, but not in the abnormal, discussed below.

When the consequences of depression were investigated .... post-depressives scored lower in verbal memory, a neuropsychological measure of hippocampal function, suggesting that the volume loss was related to an aspect of cognitive functioning [21].

This finding of decreased verbal function - memory, fluency and learning in LEI - is just what documentation of the injury indicates, along with a broader cognitive deficit also seen. This deficit is said to be the case in $50 \%$ of depressed patients, and of these those with hippocampal atrophy were especially likely to suffer from a subtype of depression with the deficits seen above and proportional to cases where cortisol oversecretion was most marked [19, 21].

The hippocampal atrophy seen is thought to be a result of either inhibition of nerve cell growth or toxic destruction of nerve cells [23]. The loss is also seen in Cushings Syndrome, where there is an over secretion of cortisol.

Thus excess of cortisol (and this is unquestionnably the case in depression resulting from stress) appears to damage neurons in the hippocampus, negatively affecting long-term potentiation of neurons, and showing as memory, cognition, and learning deficits [24].

Further, the implication of cortisol in depression and the loss of hippocampal volume seems undoubted, and this is a "new" proposal in the theory of depression.

The question arises that if cortisol destroys nerve cells, why does the brain's plastic protectivity "rescue" it by mechanism of BDNF (It has also been shown in doubleblind studies that anti-cortisol agents can act as antidepressants if the patient is diagnosed as one showing high cortisol [25]).

So the question now becomes whether or not stress inhibits neurogenesis of the hippocampus.

Studies show that long term potentiation (LTP) of hippocampal cells which enhances the memory and learning referred to above, are interfered with by stress, and infusion of BDNF is protective [26].

BDNF infusion gives significant protection against both brain tissue losses and spatial cognitive impairments. These findings indicate that stress is associated with cognitive deficits, and that BDNF pretreatment is protective against both cellular loss and spatial/memory impairment [27, 28]. 
While BDNF production parallels cortisol production in the normal person it is interesting that its production is empirically decreased by stress, and indeed stress, in reducing BDNF, deprives the brain of its plastic ability to adapt to stress [15]. That is, it is a key feature of the link between BDNF and depression that there is a lack of parallel relationship between BDNF and cortisol, and a marked negative proportionality (increased cortisol and decreased BDNF) which alters the balance between the two negatively.

But only in some.

In attempting to explain this variability, it is known that there is a genetic polymorphism of BDNF. One form of BDNF seems to be highly correlated with the production of stress related depression with the alternate is protective. The "harmful" form is associated with dysregulation of the HPA axis and the decoupling of cortisol and BDNF thus depriving the brain of its plasticity $[29,30]$. The polymorphism has been investigated. There is a gender difference and also an individual difference within sexes of the presence of each polymorphic form. Thus there is a difference in predisposition to depression fromm the same stress dependant on the polymorphism of BDNF [31].

One writer has proposed a difference in stress response between the amygdala and the hippocampus [32].

\section{CONCLUSION - AN HYPOTHESIS FOR ELI}

The author collects these disparate findings together to form a hypothesis for the production of the remote symptoms in ELI.

It is entirely reasonable to consider ELI a highly stressful event and thus it is entirely reasonable that it is productive of an intense cortisol response. That response is maintained by ongoing pain and dysfunction over some weeks, remembering that the experimental observations above were generated in animals subject to stress for as little as seven days.

In a short time, excess cortisol has a cytotoxic effect in the hippocampus. The consequence is further dysregulation of the HPA axis maintaining the state.

The further consequence is hippocampal atrophy, remembering that the hippocampus is strongly responsible for the learning and memory deficits associated with ELI.

There is a delay in onset of the neuropsychological picture, and this theory accounts for that fact.

One enigmatic feature is that only some people, even if subject to roughly the same stress, will demonstrate the Post Electric Shock Syndrome. This may be accounted for by the observation of the polymorphism of the BDNF gene, and the fact that there is a predisposition to the syndrome in only some who have the dysmorphism.

The disorder, once commenced is self perpetuating, and experience with the injury indicated that the disability lasts for a long period, and rarely if ever completely resolves. This si commensurate with the view of some that the atrophy and cellular loss is never completely reversible.

These observations lead the author to a firmer basis for experiments to test the hypothesis in ELI and for the rational use of antidepressants, and also more novel agents mentioned in the relevant literature.

The author admits that this hypothesis is quite speculative, but draws some support from the study of Kurtulus et al. [33]. In that study the authors subjected rats to electric shock with household electricity and found statistically significant loss of neurones in the hippocampus of their rats on day 3 post shock.

\section{ACKNOWLEDGEMENT}

Declared none.

\section{CONFLICT OF INTEREST}

Declared none.

\section{REFERENCES}

[1] Andrews C. SPECT Scanning and neuropsychological testing in electrical injuries. Proc ICOLSE; Seattle: Boeing Corp 2007.

[2] Andrews C. Cluster analysis of functional injuries in electrical accidents: Neuropsychological testing and functional scans: In: ICOLSE; Paris: France, 2007.

[3] Cooper MA, Andrews CJ, ten Duis H. Psychological aspects of lightning injury. 9th International Conference on Atmospheric Electricity; June 15-19, St Petersburg: Russia 1992.

[4] Engelstatter G. Psychological effects of lightning strike and electric shock injuries. 3rd. Ann Conf. Light. And Elec. Shock Victims Assn; Jacksonville, NC.: Carolina Psychological Health Service 1993.

[5] Janus TJ, Barrash J. Neurologic and neurobehavioral effects of electric and lightning injuries. J Burn Care Rehabil 1996; 17(5): 409-15.

[6] Pliskin N. Neuropsychological changes following electrical injury. J Int Neuropsychol Soc 2006;12: 17-23.

[7] Primeau M, Engelstatter G, Cooper MA. Psychological sequelae of lightning injury. ICOLSE; Williamsburg, Va 1995.

[8] Primeau M, Engelstatter GH, Bares KK. Behavioral consequences of lightning and electrical injury. Semin Neurol 1995; 15(3): 27985.

[9] Reisner AD. A case of lightning injury with delayed-onset psychiatric and cognitive symptoms. Brain Inj 2006; 20(10): 1093 7 .

[10] van Zomeren AH, ten Duis HJ, Minderhoud JM, et al. Lightning stroke and neuropsychological impairment: cases and questions. J Neurol Neurosurg Psychiatr 1998; 64(6): 763-9.

[11] Cooper MA, Andrews CJ. Disability, not death, is the issue in lightning injury. Proc ICOLSE; Seattle, WA, 2005.

[12] Andrews CJ. Further documentation of remote effects of electrical injuries, with comments on the place of neuropsychological testing and functional scanning. IEEE Trans Biomed Eng 2006; 53(10): 2102-13.

[13] Morse MS, Berg JS, ten Wolde RL. Diffuse electrical injury: a study of 89 subjects reporting long-term symptomatology that is remote to the theoretical current pathway. IEEE Trans Biomed Eng 2004; 51(8): 1449.

[14] Majzoub JA. Corticotropin-releasing hormone physiology. Eur J Endocrinol 2006; 155: S71-6.

[15] Tapia-Arancibia LR, Givalois F, Arancibia LS. Physiology of BDNF: focus on hypothalamic function. Front Neuroendocrinol 2004; 25(2): 77-107.

[16] Begliuomini S. Plasma brain-derived neurotrophic factor daily variations in men: correlation with cortisol circadian rhythm. J Endocrinol 2008; 197: 429-35.

[17] Available at: http://www.allaboutdepression.com/cau_02.htm [Accessed August 2010].

[18] Bremner JD, Narayan M, Anderson ER, Staib LH, Miller HL Charney DS. Hippocampal volume reduction in major depression. Am J Psychiatry 2000; 157(1): 115-8.

[19] Sheline Y, Wang P, Gado M. Hippocampal atrophy in recurrent major depression. Proc Natl Acad USA 1996; 93: 3908-4003.

[20] Videbach P, Ravnkilde B. Hippocampal volume and depression: A Meta-Analysis of MRI studies. Am J Psychiatry 2004; 161: 195766. 
[21] Sheline Y, Sanghavi M, Mintun M, Gado M. Depression duration but not age predicts hippocampal volume loss in medically healthy women with recurrent major depression. J Neurosci 1999; 19: 5034-41.

[22] Czeh B, Michaelis T, Watanabe T, et al. Stress Induced Changes in cerebral metabolites, hippocampal volume, and cell proliferation are prevented by antidepressant treatment with tianeptine. Proc Natl Acad Sci USA 2001; 98(22): 12796-801.

[23] Sapolsky RM. Depression, antidepressants, and the shrinking hippocampus. Proc Natl Acad Sci USA 2001; 98(22): 12320-2.

[24] Pavlides C, Nivon L, McEwen BS. Effects of chronic stress on hippocampal long-term potentiation. Hippocampus 2002; 12(2): 245-57.

[25] Wolkowitz O, Reus V. Treatment of depression with antiglucocorticoid drugs. Psychosom Med 1999; 61(5): 698-711.

[26] Radecki DT, Brown LM, Martinez J, Teyler TJ. BDNF Protects against stress induced impairments in spatial learning and memory and LTP. Hippocampus 2005; 15(2): 246-53.

[27] Almli CR, Levy TJ, Han BH, Shah AR, Gidday JM, Holtzman DM. BDNF protects against spatial memory deficits following neonatal Hypoxia-Ischaemia. Exp Neurol 2000; 166(1): 99-114.
[28] Available at: http://www.reproductive-revolution.com/archive/str ess.html [Accessed: 4 July 2010].

[29] Schule C, Zill P, Baghai TC, et al. Brain derived neurotrophic Val66Met polymorphism and dexamethasone/CRH test result in depressed patients. Psychoneuroendocrinology 2006; 31(8): 101925.

[30] Alexander N, Osinsky R, Schmitz A, et al. The BDNF Val66Met polymotphism affects HPA-axis reactivity to acute stress. Psychoneuroendocrinology 2010; 35(6): 949-53.

[31] Shalev I, Lerer E, Israel S, et al. BDNF Val66Met polymorphism is associated with HPA axis reactivity to psychological stress characterized by genotype and gender interactions. Psychoneuroendocrinology 2009; 34(3): 382-8.

[32] Vyas A, Mitra R, Shankatanarayana R, Chattarji S. Chronic Stress induces contrasting patterns of dendritic remodeling in hippocampal and amygdaloid neurons. J Neurosci 2002; 22(15): 6810-8.

[33] Kurtulus A, Acar K, Adiguzel E, Boz B. Hippocampal neuron loss due to electric injury in rats: A stereological study. Leg Med (Tokyo) 2008; 22(12): 2671-5. 